\title{
Are Neutral Minima Stable against Charge Breaking in the Higgs Triplet Model?
}

\author{
P. M. Ferreira ${ }^{1,2}$ and B. L. Gonçalves ${ }^{12,3}$ \\ ${ }^{1}$ Instituto Superior de Engenharia de Lisboa, Instituto Politécnico de Lisboa 1959-007 Lisboa, Portugal \\ ${ }^{2}$ Centro de Física Teórica e Computacional, Faculdade de Ciências, Universidade de Lisboa, Campo Grande, \\ Edifício C8 1749-016 Lisboa, Portugal \\ ${ }^{3}$ Departamento de Física and CFTP, Instituto Superior Técnico, Universidade de Lisboa, 1049-001 Lisboa, Portugal
}

\begin{abstract}
In the Higgs triplet model, it is a priori possible to have charge breaking minima developing. We analyse the possibility of such minima to be deeper than neutral ones. Analytical expressions relating the depth of minima of different types are obtained. A global symmetry of the model leads to increased stability for charge-preserving vacua. However, when a soft-breaking term is present, deeper charge-breaking minima may occur more easily. We realise that vacuum configurations with a vevless doublet can be possible, with such vev configurations changing the stability picture. We scan the model's parameter space and realise what vev configurations are most likely to produce charge breaking minima. We also obtain a necessary and sufficient condition for stability against charge-breaking vacua for the model with an intact global symmetry. ${ }^{2}$
\end{abstract}

Keywords: Higgs triplet model, neutral minima, charge breaking minima, vacuum stability

DOI: 10.31526/ACP.BSM-2021.14

\section{INTRODUCTION}

One of the fundamental pieces of the Standard Model (SM) of particle physics is the Higgs mechanism, through which elementary particles acquire mass from the spontaneous breaking of gauge symmetries, when a scalar doublet develops a non-vanishing vacuum expectation value (vev). This mechanism implies the existence of an elementary spin- 0 particle, the Higgs boson, which was finally discovered in 2012 by the LHC collaborations [2, 3]. Further measurements of the properties of this particle (see, for instance, $[4,5]$ ) show that it behaves in a very similar manner to the SM Higgs particle. However, there are still a lot of room for theories with extended scalar sectors being able to accommodate such particle. One of the most popular and simplest extensions of the SM scalar sector is the two-Higgs doublet model (2HDM), proposed by Lee in 1973 [6]. For a 2HDM review, see [7].

The Higgs-triplet model (HTM) $[8,9,10,11,12,13]$ stands as another popular theory with an extended scalar sector - a scalar triplet, with hypercharge $Y=2$, is added to the usual SM Higgs doublet. It has a richer scalar spectrum, which includes two $\mathrm{CP}$-even scalars, $h$ and $H$, a pseudoscalar $A$, a charged scalar $H^{ \pm}$and a doubly charged one, $H^{ \pm \pm}$. One of the main motivations of the HTM is the possibility of explaining the smallness of neutrino masses via a type-II seesaw mechanism. The Higgs-triplet model can also accommodate dark matter candidates, and boast a rich phenomenology.

There is an extensive body of work on the HTM (see for instance $[14,15,16,17,18,19]$ ), both on theoretical and phenomenological aspects. In this work we perform an in-depth analysis of the vacuum structure of the model, using techniques developed to study the 2HDM. In particular, we are interested in the possibility of charge-breaking (CB) vacua developing- since solutions of the minimization conditions of the potential which include vevs possessing electrical charge, which spontaneously breaks the electromagnetic symmetry, are a priori possible. With this analysis we wish to provide a tool which allows to limit the model's allowed parameter space, increasing its predictive power. Several authors [14, 15, 19] have performed numerous and distinctive analysis on the HTM vacua. However, with this work, we go further and perform the most exact and generic analysis of the HTM vacuum so far in the literature.

This manuscript is organized as follows: we briefly describe the model in section 2, with emphasis on the scalar sector and possible vacua. In section 3 we discuss the vacuum structure of the potential without the soft-breaking term. Then, in section 4 we will allow for the presence of a global symmetry soft breaking term, and show how it changes the stability of neutral vacua with respect to charge breaking. In section 5 we will study the situation where the doublet has no vev and the triplet possesses charge breaking vevs, and show how this changes the stability status we have come up with. We will end with a brief yet insightful numerical study of the model, in section 6, searching for regions where deeper CB vacua might occur, and draw some final conclusions in section 7.

\footnotetext{
${ }^{1}$ Speaker

${ }^{2}$ This contribution is based on the work of [1].
} 


\section{THE HIGGS-TRIPLET MODEL}

The Higgs-triplet model (HTM) is an extension of the scalar sector of the Standard Model (SM), which is complemented by a hypercharge 2 triplet, $\Delta$, besides the usual hypercharge 1 Higgs doublet of the SM, $\Phi$. Such fields can be written as

$$
\Phi=\left(\begin{array}{c}
\phi^{+} \\
\phi^{0}
\end{array}\right), \Delta=\left(\begin{array}{cc}
\Delta^{+} / \sqrt{2} & \Delta^{++} \\
\Delta^{0} & -\Delta^{+} / \sqrt{2}
\end{array}\right)
$$

in which all $\phi^{x}, \Delta^{y}$ are complex fields, and we use an $S U(2)$ matrix representation for the triplet $\Delta$. The most general $S U(2) \times U(1)$ scalar potential involving these two fields is then given by

$$
\begin{aligned}
V & =m^{2} \Phi^{\dagger} \Phi+M^{2} \operatorname{Tr}\left(\Delta^{\dagger} \Delta\right)+\mu\left(\Phi^{T} \mathrm{i} \tau_{2} \Delta^{\dagger} \Phi+\text { h.c. }\right) \\
& +\lambda_{1}\left(\Phi^{\dagger} \Phi\right)^{2}+\lambda_{2}\left[\operatorname{Tr}\left(\Delta^{\dagger} \Delta\right)\right]^{2}+\lambda_{3} \operatorname{Tr}\left[\left(\Delta^{\dagger} \Delta\right)^{2}\right]+\lambda_{4}\left(\Phi^{\dagger} \Phi\right) \operatorname{Tr}\left(\Delta^{\dagger} \Delta\right)+\lambda_{5} \Phi^{\dagger} \Delta \Delta^{\dagger} \Phi,
\end{aligned}
$$

with all parameters in the potential being real, and h.c. standing for "hermitian conjugate", as usual. So that the model is bounded from below - and therefore possesses a stable global minimum - the quartic couplings $\lambda_{1, \ldots 5}$ must obey the following necessary and sufficient conditions [14]:

$$
\begin{gathered}
\lambda_{1}>0, \quad \lambda_{2}+\min \left(\lambda_{3}, \lambda_{3} / 2\right)>0 \\
\lambda_{4}+\min \left(0, \lambda_{3}\right)+2 \min \left[\sqrt{\lambda_{1}\left(\lambda_{2}+\lambda_{3}\right)}, \sqrt{\lambda_{1}\left(\lambda_{2}+\lambda_{3} / 2\right)}\right]>0 .
\end{gathered}
$$

In ref. [14] bounds on the quartic couplings of the potential so that the theory preserves unitarity were also presented.

The term cubic in the fields, with coefficient $\mu$, can be removed by imposing on the potential, for instance, a global $U(1)$ symmetry of the form $\Phi \rightarrow e^{i \theta} \Phi$, with $\theta$ an arbitrary real number. Therefore, the $\mu$ term is a soft breaking of this global symmetry. The theory without this term, with the global symmetry intact, is phenomenologically interesting, since it allows for dark matter particles. On the other hand, softly breaking this continuous global symmetry is also of interest, since it can be used to help generate neutrino masses via the seesaw mechanism. Both theories are relevant, and we will study their vacuum structure separately.

A priori there are three different vacuum possibilities: neutral, CP-breaking and charge-breaking vacua. However, spontaneous $\mathrm{CP}$ breaking, in which complex neutral vevs could arise, is not possible in the Higgs-triplet model. This can be demonstrated in a remarkably simple way (as we have done in [1]). Thus we will address real neutral and charge-breaking vacua.

\subsection{The neutral vacua}

The Higgs-triplet model has three different possible minima wherein the vevs are neutral and electric charge conservation holds, each yielding very different phenomenologies. We call these Normal minima, and the three different possibilities are the following:

- The N1 stationary point, where both scalar fields have neutral vevs,

$$
\langle\Phi\rangle_{N 1}=\frac{1}{\sqrt{2}}\left(\begin{array}{c}
0 \\
v_{\Phi}
\end{array}\right),\langle\Delta\rangle_{N 1}=\frac{1}{\sqrt{2}}\left(\begin{array}{cc}
0 & 0 \\
v_{\Delta} & 0
\end{array}\right) .
$$

In order to have the correct electroweak symmetry breaking one would need to have $v_{\Phi}^{2}+2 v_{\Delta}^{2} \simeq(246 \mathrm{GeV})^{21}$. This extremum can occur whether the soft breaking $\mu$ term is present or not. Defining the quantity

$$
M_{\Delta}^{2} \equiv \frac{v_{\Phi}^{2} \mu}{\sqrt{2} v_{\Delta}}
$$

the pseudoscalar, singly charged and doubly charged scalar masses are given by

$$
\begin{gathered}
m_{A}^{2}=M_{\Delta}^{2}\left(1+4 v_{\Delta}^{2} / v_{\Phi}^{2}\right) \\
m_{+}^{2}=\left(M_{\Delta}^{2}-\lambda_{5} v_{\Phi}^{2} / 4\right)\left(1+2 v_{\Delta}^{2} / v_{\Phi}^{2}\right) \quad, \quad m_{++}^{2}=M_{\Delta}^{2}-v_{\Delta}^{2} \lambda_{3}-\lambda_{5} v_{\Phi}^{2} / 2 .
\end{gathered}
$$

If $\mu=0$ we will have $M_{\Delta}=0$ and consequently $m_{A}=0-$ the triplet vev spontaneously breaks a global continuous symmetry and the theory develops a massless axion. As for the CP-even scalars $h$ and $H$, their squared masses will be the eigenvalues of the $2 \times 2$ matrix

$$
\left[m_{h, H}^{2}\right]=\left(\begin{array}{cc}
2 \lambda_{1} v_{\phi}^{2} & -\frac{2 v_{\Delta}}{v_{\phi}} M_{\Delta}^{2}+\left(\lambda_{4}+\lambda_{5}\right) v_{\phi} v_{\Delta} \\
-\frac{2 v_{\Delta}}{v_{\phi}} M_{\Delta}^{2}+\left(\lambda_{4}+\lambda_{5}\right) v_{\phi} v_{\Delta} & M_{\Delta}^{2}+2\left(\lambda_{2}+\lambda_{3}\right) v_{\Delta}^{2}
\end{array}\right)
$$

\footnotetext{
${ }^{1}$ In the HTM, the tree-level prediction for the electroweak precision constraint parameter $\rho$ is not equal to 1 , unlike models with an arbitrary number of doublets. This then forces the triplet vev to be limited in magnitude, typically no more than $8 \mathrm{GeV}$.
} 
- The $N 2$ stationary point, where only the doublet has a vev,

$$
\langle\Phi\rangle_{N 2}=\frac{1}{\sqrt{2}}\left(\begin{array}{l}
0 \\
v
\end{array}\right),\langle\Delta\rangle_{N 2}=\frac{1}{\sqrt{2}}\left(\begin{array}{ll}
0 & 0 \\
0 & 0
\end{array}\right) .
$$

In this case, to obtain the correct electroweak symmetry breaking, one must have $v=246 \mathrm{GeV}$. Unlike N1, this extremum can only exist if $\mu=0$. The two neutral states emerging from the doublet ( $H$ and $A$ ) will be degenerate and are good dark matter candidates. The scalar masses are given by

$$
\begin{aligned}
m_{h}^{2}=2 \lambda_{1} v_{\Phi}^{2} & , \quad m_{H}^{2}=m_{A}^{2}=M^{2}+\frac{1}{2}\left(\lambda_{4}+\lambda_{5}\right) v_{\Phi}^{2}, \\
m_{+}^{2}=M^{2}+\frac{1}{4}\left(2 \lambda_{4}+\lambda_{5}\right) v_{\Phi}^{2} & , \quad m_{++}^{2}=M^{2}+\frac{1}{2} \lambda_{4} v_{\Phi}^{2}
\end{aligned}
$$

in which the SM-like Higgs boson is the $h$ scalar state.

- The N3 stationary point, where only the triplet field acquires a vev,

$$
\langle\Phi\rangle_{N 3}=\frac{1}{\sqrt{2}}\left(\begin{array}{l}
0 \\
0
\end{array}\right),\langle\Delta\rangle_{N 3}=\frac{1}{\sqrt{2}}\left(\begin{array}{cc}
0 & 0 \\
v_{\Delta} & 0
\end{array}\right) .
$$

This extremum is clearly unphysical - quarks would be massless, for instance. Therefore, we will want to avoid this vacuum if possible. Notice that N3 is a possible solution to the minimization conditions whether the soft breaking term $\mu$ is present or not. Since the masses at N3 will not be required for our stability analysis, we do not present them.

The neutral minima of greater interest for the softly broken potential is clearly N1 - in that case N2 cannot occur and an N3 minimum would imply massless quarks. On the other hand, if the potential has a global continuous symmetry that is not softly broken, then it is $\mathrm{N} 2$ the neutral minimum that is relevant for particle physics phenomenology - N1 would imply a massless axion, and $N 3$ is, once again, unphysical.

\subsection{The charge breaking vacua}

For charge breaking (CB) to occur one or more vevs carrying electrical charge need to appear due to spontaneous symmetry breaking. Such vevs would generate a non-vanishing photon mass and would spoil the observed behaviour of electromagnetism. Both the doublet and triplet fields have charged components, so there is a plethora of possible CB extrema.

Via a suitable gauge choice, it is always possible to absorb three real scalar component fields. We choose to be analogous to the SM unitary gauge with the doublet being reduced to a neutral, real component. As such, and without loss of generality, in all considered field vevs, the doublet vev is always real and neutral. In the following, we will only be considering configurations with real vevs. The reader can check the full work on [1], in which we show the results obtained for CB vevs with imaginary parts. Such imaginary vevs do not bring any new features that cannot be established by looking at real vevs alone. With this in mind, there are six possible $C B$ vev choices, $C B 1$ through $C B 6$ :

$$
\begin{aligned}
& \langle\Phi\rangle_{C B 1}=\frac{1}{\sqrt{2}}\left(\begin{array}{c}
0 \\
c_{1}
\end{array}\right) \quad, \quad\langle\Delta\rangle_{C B 1}=\frac{1}{\sqrt{2}}\left(\begin{array}{cc}
-c_{3} / \sqrt{2} & 0 \\
c_{2} & c_{3} / \sqrt{2}
\end{array}\right) \\
& \langle\Phi\rangle_{C B 2}=\frac{1}{\sqrt{2}}\left(\begin{array}{c}
0 \\
c_{1}
\end{array}\right) \quad, \quad\langle\Delta\rangle_{C B 2}=\frac{1}{\sqrt{2}}\left(\begin{array}{cc}
0 & c_{3} \\
c_{2} & 0
\end{array}\right) \\
& \langle\Phi\rangle_{C B 3}=\frac{1}{\sqrt{2}}\left(\begin{array}{c}
0 \\
c_{1}
\end{array}\right) \quad, \quad\langle\Delta\rangle_{C B 3}=\frac{1}{\sqrt{2}}\left(\begin{array}{cc}
c_{3} / \sqrt{2} & c_{4} \\
c_{2} & -c_{3} / \sqrt{2}
\end{array}\right) \\
& \langle\Phi\rangle_{C B 4}=\frac{1}{\sqrt{2}}\left(\begin{array}{c}
0 \\
c_{1}
\end{array}\right) \quad, \quad\langle\Delta\rangle_{C B 4}=\frac{1}{\sqrt{2}}\left(\begin{array}{cc}
c_{2} / \sqrt{2} & 0 \\
0 & -c_{2} / \sqrt{2}
\end{array}\right) \\
& \langle\Phi\rangle_{C B 5}=\frac{1}{\sqrt{2}}\left(\begin{array}{c}
0 \\
c_{1}
\end{array}\right) \quad, \quad\langle\Delta\rangle_{C B 5}=\frac{1}{\sqrt{2}}\left(\begin{array}{cc}
c_{2} / \sqrt{2} & c_{3} \\
0 & -c_{2} / \sqrt{2}
\end{array}\right) \\
& \langle\Phi\rangle_{C B 6}=\frac{1}{\sqrt{2}}\left(\begin{array}{c}
0 \\
c_{1}
\end{array}\right) \quad, \quad\langle\Delta\rangle_{C B 6}=\frac{1}{\sqrt{2}}\left(\begin{array}{cc}
0 & c_{2} \\
0 & 0
\end{array}\right)
\end{aligned}
$$

The CB3 case is clearly the most generic vev pattern possible. The remaining cases correspond to different possibilities, $a$ priori allowed by the minimisation conditions of the potential. Let us emphasise that the quantities $c_{1}, \ldots c_{4}$ are not supposed to be equal for different $\mathrm{CB}$ extrema. For each of the $\mathrm{CB}$ cases considered, the value of the correspondent vevs $c_{i}$ must be determined by the minimisation of the potential, and depend on the model's parameters. In the following two sections, we will also consider $c_{1} \neq 0$ - the doublet will always have a non-vanishing vev. 


\section{POTENTIAL WITHOUT SOFT-BREAKING TERM}

If a global continuous symmetry is imposed on the potential, the $\mu$ term in Eq. (2.2) is zero. There are no cubic terms in the potential, then, only quadratic, $V_{2}$, and quartic, $V_{4}$, ones. Thus, the potential $V$ can be written as $V=V_{2}+V_{4}$. Any solution of the minimisation equations of the potential will imply a simple relation between the values of $V_{2}$ and $V_{4}$ at any stationary point. Since the potential only has quadratic and quartic terms when $\mu=0$, and we are interested in comparing the value of the potential at different extrema, it is tempting to attempt to use a bilinear formalism similar to the one employed for the 2HDM [20, 21, 22, 23, 24, $25,26,27,28,29,30,31,32,33]$. The major problem in attempting the formulation of a bilinear formalism for this model is concerned with the $\lambda_{3}$ and $\lambda_{5}$ terms in the scalar potential, which cannot obviously be written as the product of two terms quadratic in the fields. This would seem to be an unsurmountable obstacle to a bilinear formulation but, in fact, can easily be overcomed. For the study of the vacuum structure of the scalar potential, and the comparison of the value of that potential at different extrema, we do not need the full field-dependent potential, but rather only the potential as a function of the vevs at the several stationary points. In [1], we explicitly present how such formulation is applied to the Higgs-triplet model, with several instructive and explicit examples.

As we have stated, without the soft breaking term, the N2 minimum is the most phenomenologically appealing. We can thus apply our bilinear formalism to any pairs of extrema N2 versus CB, and obtain the expressions relating the depth of $N 2$ relative to any of the six CB configurations, written as a function of the $c_{i}$ vevs and the $N 2$ scalar quadratic masses. That same formalism can also be adapted to N1 stationary points, giving rise to analytical expressions relating the depth of the potential at $N 1$ and $\mathrm{CB}$ extrema as functions of the $c_{i}$ vevs and the scalar masses now evaluated at an N1 extremum. The results for both minima are joined together in Table 1.

\begin{tabular}{|c|c|c|}
\hline & \multicolumn{2}{|c|}{$\mu=0$ and $c_{1} \neq 0$} \\
\hline & N2 & 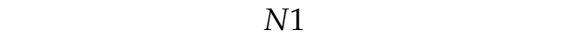 \\
\hline$V_{C B 1}-V_{N i}$ & $\frac{1}{4}\left(c_{2}^{2} m_{H, A}^{2}+c_{3}^{2} m_{+}^{2}\right)$ & $\frac{c_{3}^{2} m_{+}^{2}}{4\left(1+\frac{2 v_{\Delta}^{2}}{v_{\Phi}^{2}}\right)}$ \\
\hline$V_{C B 2}-V_{N i}$ & $\frac{1}{4}\left(c_{2}^{2} m_{H, A}^{2}+c_{3}^{2} m_{++}^{2}\right)$ & $\frac{1}{4} c_{3}^{2} m_{++}^{2}$ \\
\hline$V_{C B 3}-V_{N i}$ & $\frac{1}{4}\left(c_{2}^{2} m_{H, A}^{2}+c_{3}^{2} m_{+}^{2}+c_{4}^{2} m_{++}^{2}\right)$ & $\frac{m_{+}^{2} c_{3}^{2}}{4\left(1+\frac{2 v_{\Delta}^{2}}{v_{\Phi}^{2}}\right)}+\frac{1}{4} c_{4}^{2} m_{++}^{2}-\frac{1}{8} \lambda_{3} v_{\Delta}^{2} \frac{c_{3}^{2} c_{4}}{c_{2}}$ \\
\hline$V_{C B 4}-V_{N i}$ & $\frac{1}{4} c_{2}^{2} m_{+}^{2}$ & $\frac{c_{1}^{2} m_{+}^{2}}{4\left(2+\frac{v_{\Phi}^{2}}{v_{\Delta}^{2}}\right)}+\frac{1}{8} c_{2}^{2} m_{++}^{2}$ \\
\hline$V_{C B 5}-V_{N i}$ & $\frac{1}{4}\left(c_{2}^{2} m_{+}^{2}+c_{3}^{2} m_{++}^{2}\right)$ & $\frac{c_{1}^{2} m_{+}^{2}}{4\left(2+\frac{v_{\Phi}^{2}}{v_{\Delta}^{2}}\right)}+\frac{1}{8} c_{2}^{2} m_{++}^{2}+\frac{c_{3}^{2} m_{+}^{2}}{2\left(1+\frac{2 v_{\Delta}^{2}}{v_{\Phi}^{2}}\right)}$ \\
\hline$V_{C B 6}-V_{N i}$ & $\frac{1}{4} c_{2}^{2} m_{++}^{2}$ & $\frac{c_{1}^{2} m_{+}^{2}}{2\left(2+\frac{v_{\Phi}^{2}}{v_{\Delta}^{2}}\right)}+\frac{c_{2}^{2} m_{+}^{2}}{2\left(1+\frac{2 v_{\Delta}^{2}}{v_{\Phi}^{2}}\right)}$ \\
\hline
\end{tabular}

TABLE 1: Expressions relating the depth of the potential at $N i$ and CB, with $i=1,2$.

As we see, for all possible cases, when $N 2$ is a minimum one always obtains $V_{C B i}-V_{N 2}>0$. This holds even if one considers complex charge breaking vevs [1], and thus the stability of $N 2$ against charge breaking seems to be guaranteed - provided that $c_{1} \neq 0$.

When considering the $N 1$ case, since all of its squared masses will be positive, all the potential differences have positive values, except for the CB3 extrema. In this case, the simultaneous occurrence of both $N 1$ and $C B 3$ extrema is only possible if $\lambda_{3}=\lambda_{5}=0$, which implies $m_{+}^{2}=m_{++}^{2}=0$. This leads to the degeneracy of both extrema, $V_{C B 3}-V_{N 1}=0$. This of course means that such coexistence of extrema implies that for such parameter choices CB3 ceases to be charge-breaking ${ }^{2}$.

The upshot is that for generic scalar potential parameters where neither $\lambda_{3}$ nor $\lambda_{5}$ are zero, there will be no CB3 extrema coexisting with N1. Thus N1 minima are stable against the possibility of deeper charge breaking minima occurring. This conclusion, like for the $N 2$ case, also holds if one considers complex CB vevs. We have again assumed that in the CB vacua the doublet has a non-vanishing vev, $c_{1} \neq 0$. Relaxing this assumption will significantly change these conclusions.

\footnotetext{
${ }^{2}$ In fact, without the $\lambda_{3}$ and $\lambda_{5}$ terms in the potential, it becomes possible to perform two independent $S U(2)$ transformations on the doublet and triplet, and thus "rotate away" the charge breaking vevs of the triplet, transforming a seeming CB3 vacuum into an N1 one.
} 


\section{POTENTIAL WITH SOFT-BREAKING TERM}

The introduction of the soft breaking term $\mu$ changes many things. Phenomenologically, the $N 2$ vacuum ceases to be possible - the minimisation conditions have no solution with $v_{\Delta}=0$ when $v_{\phi} \neq 0$. The $N 1$ vacuum no longer implies a massless scalar, rather that state has a mass directly proportional to $\mu$. Regarding the stability of neutral vacua, the soft breaking term has a significant impact . Let us begin by recalling that the $\mu$ term in the potential is cubic in the fields. The potential is therefore no longer a sum of quadratic and quartic terms as previously, but it has a new cubic contribution. Now, such cubic terms in the vevs mean that the application of the bilinear formalism is not at all obvious or straightforward, since it relies on quadratic-plus-quartic potentials being easily expressed as polynomials of quadratic field/vev variables. Nevertheless, with some ingenuity, we can follow the steps outlined for the non soft breaking cases and adapt the demonstrations to include the cubic terms when necessary. In [1], we explicitly perform one of the calculations relating the value of the potential at two pairs of extrema, obtaining the first case relating N1 with CB1:

$$
V_{C B 1}-V_{N 1}=\frac{m_{A}^{2}}{4\left(1+\frac{4 v_{\Delta}^{2}}{v_{\Phi}^{2}}\right)}\left(c_{2}-v_{\Delta}\right)^{2}\left(1-\frac{v_{\Delta}}{c_{2}} \frac{c_{1}^{2}}{v_{\Phi}^{2}}\right)+\frac{m_{+}^{2} c_{3}^{2}}{4\left(1+\frac{2 v_{\Delta}^{2}}{v_{\Phi}^{2}}\right)}
$$

As before, the difference in the values of the potentials at a CB stationary point and a N1 one can be expressed as a function of vevs and the squared masses at $N 1$. Taking the limit $\mu \rightarrow 0$ in this expression (equivalent to put $m_{A}=0$ ) one recovers the non-soft breaking expression we can find in Table 1 . Unlike the $\mu=0$ case, however, now even if $N 1$ is a minimum, rendering both $m_{A}^{2}$ and $m_{+}^{2}$ positive, it is no longer guaranteed that $V_{C B 1}-V_{N 1}>0$. The reason is the coefficient $-v_{\Delta} / c_{2}$, which opens up the possibility of having $V_{C B 1}-V_{N 1}<0$ even if $N 1$ is a minimum.

Thus, regarding $C B 1$, the soft breaking coefficient $\mu$ completely changes the stability properties of the $N 1$ minimum. It is possible to find the remaining expressions for $N 1$ coexisting with other $C B$ extrema:

$$
\begin{aligned}
& V_{C B 2}-V_{N 1}=\frac{m_{A}^{2}}{4\left(1+\frac{4 v_{\Delta}^{2}}{v_{\Phi}^{2}}\right)}\left(c_{2}-v_{\Delta}\right)^{2}\left(1-\frac{v_{\Delta}}{c_{2}} \frac{c_{1}^{2}}{v_{\Phi}^{2}}\right)+\frac{1}{4} c_{3}^{2} m_{++}^{2} \\
& V_{C B 3}-V_{N 1}=\frac{m_{A}^{2}}{4\left(1+\frac{4 v_{\Delta}^{2}}{v_{\Phi}^{2}}\right)}\left(c_{2}-v_{\Delta}\right)^{2}\left(1-\frac{v_{\Delta}}{c_{2}} \frac{c_{1}^{2}}{v_{\Phi}^{2}}\right)+\frac{m_{+}^{2} c_{3}^{2}}{4\left(1+\frac{2 v_{\Delta}^{2}}{v_{\Phi}^{2}}\right)}+\frac{1}{4} c_{4}^{2} m_{++}^{2}-\frac{1}{8} \lambda_{3} v_{\Delta}^{2} \frac{c_{3}^{2} c_{4}}{c_{2}} \\
& V_{C B 4}-V_{N 1}=\frac{m_{A}^{2}}{4\left(1+\frac{4 v_{\Delta}^{2}}{v_{\Phi}^{2}}\right)}\left(\frac{c_{2}^{2}}{2}+v_{\Delta}^{2}+c_{1}^{2} \frac{v_{\Delta}^{2}}{v_{\Phi}^{2}}\right)+\frac{1}{8} c_{2}^{2} m_{++}^{2}+\frac{v_{\Delta}^{2}}{v_{\Phi}^{2}} \frac{c_{1}^{2} m_{+}^{2}}{4\left(1+\frac{2 v_{\Delta}^{2}}{v_{\Phi}^{2}}\right)} \\
& V_{C B 5}-V_{N 1}=\frac{m_{A}^{2}}{4\left(1+\frac{4 v_{\Delta}^{2}}{v_{\Phi}^{2}}\right)}\left(\frac{c_{2}^{2}}{2}+v_{\Delta}^{2}+c_{1}^{2} \frac{v_{\Delta}^{2}}{v_{\Phi}^{2}}-c_{3}^{2}\right)+\frac{1}{8} c_{2}^{2} m_{++}^{2}+\frac{m_{+}^{2}}{4\left(1+\frac{2 v_{\Delta}^{2}}{v_{\Phi}^{2}}\right)}\left(c_{1}^{2} \frac{v_{\Delta}^{2}}{v_{\Phi}^{2}}+2 c_{3}^{2}\right) \\
& V_{C B 6}-V_{N 1}=\frac{m_{A}^{2}}{4\left(1+\frac{4 v_{\Delta}^{2}}{v_{\Phi}^{2}}\right)}\left(v_{\Delta}^{2}-c_{2}^{2}\right)+\frac{m_{+}^{2}}{2\left(1+\frac{2 v_{\Delta}^{2}}{v_{\Phi}^{2}}\right)}\left(c_{1}^{2} \frac{v_{\Delta}^{2}}{v_{\Phi}^{2}}+c_{2}^{2}\right) .
\end{aligned}
$$

Only the CB4 case is guaranteed to give $V_{C B 4}-V_{N 1}>0$ when $N 1$ is a minimum. In all other five cases, there is always at least one term somewhere in the expressions that can render the potential differences negative even when N1 is a minimum. The inclusion of complex vevs only reinforces this conclusion [1]. Thus, we conclude that "turning on" the soft breaking term in the potential weakens the stability of neutral minima - even if $N 1$ is a minimum, there may be regions of parameter space for which deeper charge breaking vacua with $c_{1} \neq 0$ may occur.

We summarise our results in Table 2 , for both versions of the model, considering our underlying assumption $c_{1} \neq 0$.

\begin{tabular}{lcc}
\hline & \multicolumn{2}{c}{ CB minima $c_{1} \neq 0$} \\
& $\mu=0$ & $\mu \neq 0$ \\
\hline N2 minima & STABILITY GUARANTEED & DOES NOT OCCUR \\
N1 minima & STABILITY GUARANTEED & STABILITY NOT GUARANTEED \\
\hline
\end{tabular}

TABLE 2: Stability results of N2 and N1 minima against CB minima, with $c_{1} \neq 0$. 


\section{THE CASE OF THE VEVLESS DOUBLET}

Up until this point we have been considering only $\mathrm{CB}$ vev configurations with $c_{1} \neq 0$. Consider, however, the first derivative of the potential with respect to $c_{1}[1]$, which is given by

$$
\frac{\partial V}{\partial c_{1}}=c_{1}\left[m^{2}+\lambda_{1} c_{1}^{2}+\frac{\lambda_{4}}{2}\left(c_{2}^{2}+c_{3}^{2}+c_{4}^{2}\right)+\frac{\lambda_{5}}{2}\left(2 c_{2}^{2}+c_{3}^{2}\right)\right]=0 .
$$

From here we see that the trivial solution $c_{1}=0$ is always possible, regardless of the values of the parameters. This is a disconnected solution from $c_{1} \neq 0$ and the conclusions we drew for $\mathrm{CB}$ vacua with $c_{1} \neq 0$ cannot be extended to the vevless doublet case by simply considering $c_{1} \rightarrow 0$. Thus there is the possibility that the $c_{1}=0$ case brings qualitatively different conclusions, and indeed that is the case.

With $c_{1}=0$, there are six new possible real $C B$ vev configurations, $C B 7$ trough $C B 12$, for which the minimisation equations give non-trivial solutions:

$$
\begin{aligned}
& \langle\Phi\rangle_{C B 7}=\frac{1}{\sqrt{2}}\left(\begin{array}{l}
0 \\
0
\end{array}\right) \quad, \quad\langle\Delta\rangle_{C B 7}=\frac{1}{\sqrt{2}}\left(\begin{array}{cc}
c_{3} / \sqrt{2} & c_{2} \\
c_{2} & -c_{3} / \sqrt{2}
\end{array}\right) \\
& \langle\Phi\rangle_{C B 8}=\frac{1}{\sqrt{2}}\left(\begin{array}{l}
0 \\
0
\end{array}\right) \quad, \quad\langle\Delta\rangle_{C B 8}=\frac{1}{\sqrt{2}}\left(\begin{array}{cc}
0 & c_{2} \\
c_{2} & 0
\end{array}\right) \\
& \langle\Phi\rangle_{C B 9}=\frac{1}{\sqrt{2}}\left(\begin{array}{l}
0 \\
0
\end{array}\right) \quad, \quad\langle\Delta\rangle_{C B 9}=\frac{1}{\sqrt{2}}\left(\begin{array}{cc}
0 & -c_{2} \\
c_{2} & 0
\end{array}\right) \\
& \langle\Phi\rangle_{C B 10}=\frac{1}{\sqrt{2}}\left(\begin{array}{l}
0 \\
0
\end{array}\right) \quad, \quad\langle\Delta\rangle_{C B 10}=\frac{1}{\sqrt{2}}\left(\begin{array}{cc}
c_{3} / \sqrt{2} & -c_{3}^{2} / 2 c_{2} \\
c_{2} & -c_{3} / \sqrt{2}
\end{array}\right) \\
& \langle\Phi\rangle_{C B 11}=\frac{1}{\sqrt{2}}\left(\begin{array}{l}
0 \\
0
\end{array}\right) \quad, \quad\langle\Delta\rangle_{C B 11}=\frac{1}{\sqrt{2}}\left(\begin{array}{cc}
0 & c_{4} \\
0 & 0
\end{array}\right) \\
& \langle\Phi\rangle_{C B 12}=\frac{1}{\sqrt{2}}\left(\begin{array}{l}
0 \\
0
\end{array}\right) \quad, \quad\langle\Delta\rangle_{C B 12}=\frac{1}{\sqrt{2}}\left(\begin{array}{cc}
c_{3} / \sqrt{2} & 0 \\
0 & -c_{3} / \sqrt{2}
\end{array}\right)
\end{aligned}
$$

We can now apply the same methodology of previous sections to the comparison of the value of the potential at each of the above CB vacua and the normal ones.

\subsection{Stability of minima of type N1 and N2 against charge breaking without soft-breaking}

With $\mu=0$ the potential has an intact global symmetry. We have concluded that, without such soft breaking term, there was no possibility of deeper CB vacua with $c_{1} \neq 0$ than neutral ones. Now, however, this is not the case:

$$
\begin{aligned}
V_{C B 7}-V_{N 1} & =\frac{v_{\Phi}^{2}}{v_{\Delta}^{2}} \frac{m_{h}^{2} m_{H}^{2}}{16\left(\lambda_{2}+\lambda_{3}\right)}-\lambda_{3} \frac{\left[2\left(\lambda_{2}+\lambda_{3}\right) v_{\Delta}^{2}+\left(\lambda_{4}+\lambda_{5}\right) v_{\Phi}^{2}\right]^{2}}{16\left(\lambda_{2}+\lambda_{3}\right)\left(2 \lambda_{2}+\lambda_{3}\right)} \\
V_{C B 10}-V_{N 1} & =\frac{v_{\Phi}^{2}}{v_{\Delta}^{2}} \frac{m_{h}^{2} m_{H}^{2}}{16\left(\lambda_{2}+\lambda_{3}\right)} .
\end{aligned}
$$

The expression for $V_{C B 7}-V_{N 1}$ holds for $C B 8, C B 9$ and $C B 12$, while the second one holds for $V_{C B 11}-V_{N 1}$. From Eq. (5.8) we conclude that an $N 1$ minimum is stable against deeper vacua $C B 10$ (and $C B 11)^{3}$ but, even if $N 1$ is a minimum, there is no guarantee that $V_{C B 7}-V_{N 1}>0$. This completely changes the stability properties of this version of the HTM - neutral minima in a version with a global symmetry were only seemingly CB-stable since in fact deeper CB vacua with vevless doublet are possible.

For N2 minima, we have:

$$
\begin{aligned}
V_{C B 7}-V_{N 2} & =\frac{1}{4}\left(\frac{m^{4}}{\lambda_{1}}-\frac{M^{4}}{\lambda_{2}+\frac{1}{2} \lambda_{3}}\right) \\
V_{C B 10}-V_{N 2} & =\frac{1}{4}\left(\frac{m^{4}}{\lambda_{1}}-\frac{M^{4}}{\lambda_{2}+\lambda_{3}}\right)
\end{aligned}
$$

These expressions confirm that minima of type $\mathrm{N} 2$ are not guaranteed to be stable against charge breaking since deeper CB vacua with $c_{1}=0$ may well exist.

\footnotetext{
${ }^{3}$ Due to the boundedness-from-below conditions, the quantity $\lambda_{2}+\lambda_{3}$ in Eq. (5.8) is ensured to be positive.
} 


\subsection{Stability of minima of type N1 against charge breaking with soft-breaking}

Finally, considering now the case of the potential with a soft breaking term $\mu$, CB vacua with a vevless doublet can also occur, and its relationship with the vacuum $N 1$ are such that:

$$
\begin{aligned}
& V_{C B 7}-V_{N 1}=\frac{v_{\Phi}^{2}}{v_{\Delta}^{2}} \frac{m_{h}^{2} m_{H}^{2}}{16\left(\lambda_{2}+\lambda_{3}\right)}-\frac{\lambda_{1}}{8\left(\lambda_{2}+\lambda_{3}\right)} \frac{m_{A}^{2}}{1+\frac{4 v_{\Delta}^{2}}{v_{\Phi}^{2}}} \frac{v_{\Phi}^{4}}{v_{\Delta}^{2}}-\lambda_{3} \frac{\left[2\left(\lambda_{2}+\lambda_{3}\right) v_{\Delta}^{2}+\left(\lambda_{4}+\lambda_{5}\right) v_{\Phi}^{2}\right]^{2}}{16\left(\lambda_{2}+\lambda_{3}\right)\left(2 \lambda_{2}+\lambda_{3}\right)} \\
& +\frac{\lambda_{3}}{2\left(2 \lambda_{2}+\lambda_{3}\right)} \frac{m_{A}^{2}}{1+\frac{4 v_{\Delta}^{2}}{v_{\Phi}^{2}}}\left[v_{\Delta}^{2}+\frac{\lambda_{4}+\lambda_{5}}{2\left(\lambda_{2}+\lambda_{3}\right)} v_{\Phi}^{2}-\frac{1}{2\left(\lambda_{2}+\lambda_{3}\right)} \frac{m_{A}^{2}}{1+\frac{4 v_{\Delta}^{2}}{v_{\Phi}^{2}}}\right] \\
& V_{C B 10}-V_{N 1}=\frac{v_{\Phi}^{2}}{v_{\Delta}^{2}} \frac{m_{h}^{2} m_{H}^{2}}{16\left(\lambda_{2}+\lambda_{3}\right)}-\frac{\lambda_{1}}{8\left(\lambda_{2}+\lambda_{3}\right)} \frac{m_{A}^{2}}{1+\frac{4 v_{\Delta}^{2}}{v_{\Phi}^{2}}} \frac{v_{\Phi}^{4}}{v_{\Delta}^{2}}
\end{aligned}
$$

The expression for $V_{C B 7}-V_{N 1}$ holds for the cases $C B 8, C B 9$ and $C B 12$ while the second one also holds for $V_{C B 11}-V_{N 1}$. We again conclude that the fact that $N 1$ is a minimum does not guarantee its stability against deeper charge breaking vacua. Since we had already identified, in such softly-broken model, CB vacuum configurations for which deeper CB minima could coexist with neutral ones, the vevless doublet case does not bring any qualitatively different conclusions.

In Table 3, the results for the vevless doublet case are summarised, with stability not being guaranteed in any situation.

\begin{tabular}{lcc}
\hline & \multicolumn{2}{c}{ CB minima $c_{1}=0$} \\
& & $\mu \neq 0$ \\
\hline N2 minima & STABILITY NOT GUARANTEED & DOES NOT OCCUR \\
N1 minima & STABILITY NOT GUARANTEED & STABILITY NOT GUARANTEED
\end{tabular}

TABLE 3: Stability results of N2 and N1 minima against CB minima, with $c_{1}=0$.

\section{NUMERICAL ANALYSIS}

To establish the relevance of our analytical results, we will now undertake a numerical analysis of the parameter space of the Higgs Triplet Model, searching for CB minima deeper than neutral ones. We do not intent to perform an exhaustive scan of the model's parameter space. The aim here is to verify whether restrictions on the model's parameters can be obtained by requiring the global minimum of the model to be neutral, thus increasing its predictive power.

We begin our numerical analysis with the global symmetry intact, without the soft breaking term $\mu$. This model's version has a vaccum of type $\mathrm{N}^{4}$, given by Eq. (2.8), which includes possible dark matter candidates - the CP-even scalar $H$ or the pseudoscalar $A$, degenerate in mass. We generated a large sample of combinations of parameters satisfying the following conditions:

- The SM-like Higgs boson, $h$, has a mass of $125 \mathrm{GeV}$; the remaining scalar masses were chosen randomly in the intervals

$$
50 \leq m_{H}=m_{A} \leq 1000 \mathrm{GeV}, \max \left\{m_{H}, 400\right\} \leq m_{+}, m_{++} \leq 1000 \mathrm{GeV}
$$

- The quartic couplings $\lambda_{2}$ and $\lambda_{3}$ are chosen randomly and independently in the interval $[-10,10]$.

- The quadratic parameter $M^{2}$ is chosen randomly in the interval $\left[-10^{6}, 10^{5}\right] \mathrm{GeV}^{2}$.

Eqs. (2.9) relate the masses and the couplings at this N2 minimum, and allow us to fully specify all parameters of the potential. We also required that the quartic couplings obeyed the bounded from below and unitarity conditions described in section 2 . The $h$ scalar behaves, in all of its production and decay channels, very much like the SM Higgs boson, as current LHC results indicate is the case ${ }^{5}$. Once the parameter space was generated we searched for charge breaking minima. We identified the most likely $\mathrm{CB}$ vacua - in this case, the vev combinations we dubbed CB7 and CB10, as defined in Eqs. (5.2) and (5.5). We scanned the model's parameter space, looking for N2 minima and, when found any, we look if there were deeper CB ones. Check [1] for the detailed procedure employed here. Our results are depicted in Fig. 1. In the plot we present the distribution of the parameter points in the

\footnotetext{
${ }^{4}$ We have checked that, if $N 2$ is a minimum, then $N 2$ is always deeper than any $N 1$ minimum, in the version with the global symmetry intact.

${ }^{5}$ Notice that, due to the intact global symmetry, the $h$ scalar has tree-level couplings to fermions and gauge bosons identical to those of the SM. We can therefore be confident that the chosen parameter space yields a $125 \mathrm{GeV}$ scalar with properties in numerical agreement with LHC results.
} 


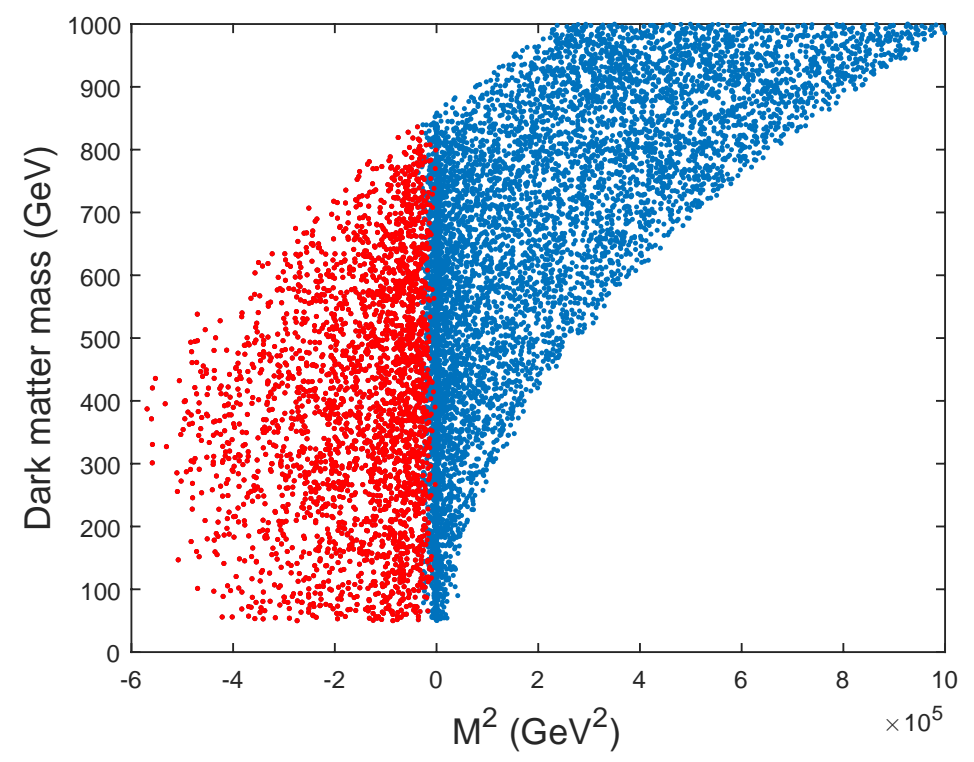

FIGURE 1: Values of the dark matter particle mass as a function of the quadratic coupling $M^{2}$ for a minimum of type N2. In blue, all the scanned points; in red, those points for which there exists a CB vacuum (of types CB7 or CB10) lower than N2.

$M^{2}-m_{H}$ plane (recall that for this minimum $H$ and $A$ have degenerate masses and are dark matter candidates). The blue points are the totality of the scan. The red points are a subset of the blue ones and indicate the regions of parameter space for which there is a $C B$ vacuum (of types $C B 7$ or $C B 10$ ) lower than the $N 2$ minimum. Some interesting features emerge from this plot. We see, for instance, that the existence of a $C B$ extremum requires a negative $M^{2}$. We also observe that $M^{2}>0$ is a sufficient condition for N2 stability, but not a necessary one.

In all, roughly $27 \%$ of the scanned parameter space includes global $C B$ vacua. Although one would need to compute the tunneling time between $N 2$ and the global minima, the simple fact that we found that such a large percentage of parameter points have a deeper $C B$ vacuum is significant. Analysing directly the expressions relating the relative depth of the potential at $N 2$ and $C B 7$ or $C B 10$, in Eqs. (5.9), we can easily deduce a necessary and sufficient condition for the non-existence of deeper $C B$ vacua. Firstly, one has to guarantee $V_{C B i}-V_{N 2}>0$. This condition together with the requirement that for all positive values of $M^{2}$, neutral vacua stability is guaranteed, and that $N 2$ is a minimum, which yields $m^{2}=-\lambda_{1} v^{2}=-m_{h}^{2} / 2$, we obtain:

$$
\text { An N2 minimum is stable against charge breaking iff } M^{2}>-\sqrt{\min \left(\lambda_{2}+\frac{1}{2} \lambda_{3}, \lambda_{2}+\lambda_{3}\right)} \frac{m_{h} v}{\sqrt{2}} \text {. }
$$

Considering now a softly broken model with a minimum of type $N 1$, the analysis of the previous sections shows there are several types of possible deeper $C B$ vacua. We scanned over the model's parameter space, allowing the triplet vev to be at most $\sim 8$ $\mathrm{GeV}$, in order to comply with electroweak precision constraints $[14,15,16,17,18]$. We allowed the quartic parameters $\left\{\lambda_{2}, \lambda_{3}, \lambda_{4}\right\}$ to vary between -10 and 10 and used the expressions for the eigenvalues of the CP-even mass matrix to, through the input of the values of $m_{h}$ and $m_{H}$, determine the quartic coupling $\lambda_{5}$ and the soft breaking parameter $\mu$. With all the potential's parameters thus established we demanded that they obeyed unitarity and boundedness from below conditions; and also that the phenomenology of the $125 \mathrm{GeV}$ scalar, $h$, be SM-like as per current LHC results. Once again, our purpose is not to perform a complete parameter space scan but rather show that CB bounds are relevant to phenomenologically appealing regions of parameter space of the model.

Having determined the full set of parameters caracterising an N1 minimum we then proceed to verify whether there is a deeper $C B$ vacuum, by performing a numerical minimisation of the potential whilst allowing the $C B$ vevs to be non-zero. Our results are shown in Fig. 2, in which we plot the doubly charged scalar mass $m_{++}$as a function of $M^{2}$. As before, in blue we represent all the scanned points, and in red the subset - a little over $48 \%$ - of those points for which there is a CB vacuum below the N1 minimum. As in the non-soft breaking $N 2$ case we see that deeper $C B$ vacua occur exclusively for $M^{2}<0$. Despite that similarity, the existence of the $\mu$ parameter changes considerably the stability picture of the neutral minima - not only there is a greater percentage of unstable $N 1$ minima for the potential which includes $\mu$, but also the regions for which $N 1$ stability is guaranteed are now quite different from the N2 case. Such a large percentage of potentially-unstable neutral minima shows that one needs to be careful when considering parameter scans of the Higgs Triplet Model, with some values of the parameters chosen actually predicting a global $C B$ minimum.

\section{CONCLUSIONS}

We have performed a deep analysis of the stability of neutral minima in the Higgs Triplet Model against the possibility of deeper charge breaking minima developing. We obtained analytical expressions relating the difference in the depths of the potential at 


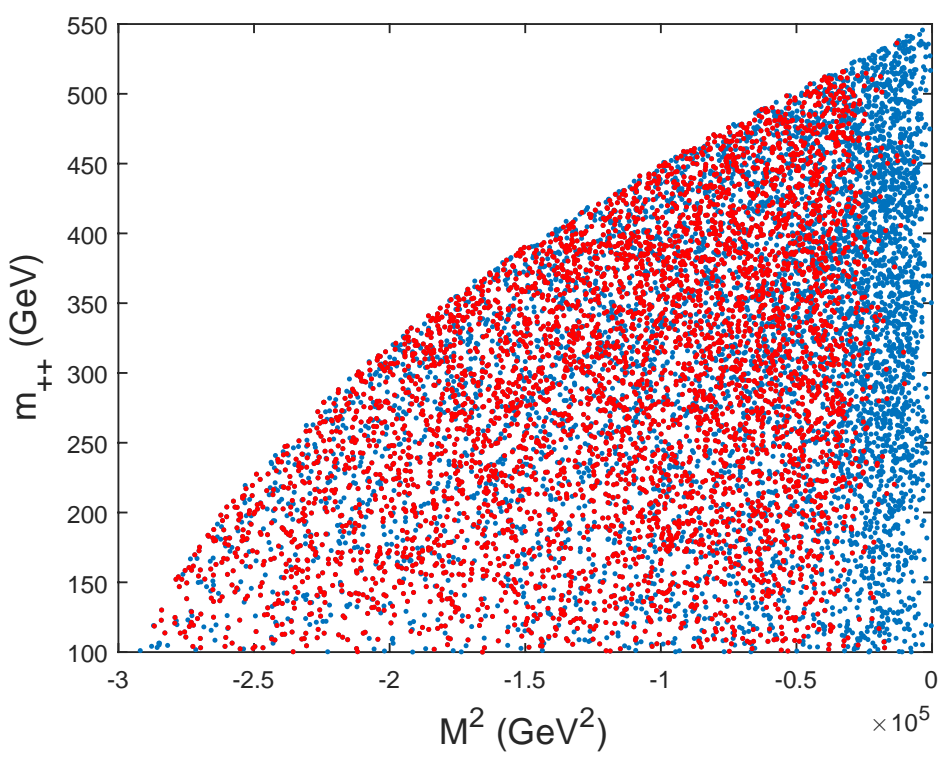

FIGURE 2: Values of the doubly charged scalar mass a function of the quadratic coupling $M^{2}$ for a minimum of type N1 in an HTM with softly broken global symmetry. In blue, all the scanned points; in red, those points for which there exists a CB vacuum (of types $C B 7$ or $C B 10$ ) lower than $N 1$.

neutral and CB extrema. Our analysis was separated in two versions of the model - the one possessing a global symmetry and the one where that same symmetry is softly broken by a cubic term. We also performed a separate study of the CB vev configurations with or without a vev for the doublet. The analytical calculations helped us establish that, in some cases, only the vevless doublet vacua could yield CB global minima. They also established that the inclusion of the soft breaking term induces more possibilities of $\mathrm{CB}$ vacua developing. To verify the relevance of $\mathrm{CB}$ bounds one might obtain we performed a numerical scan over the parameter space of the model. We found that for roughly $26 \%$ (48\%) of the parameter space found for the globally symmetric (softly broken) potential neutral minima had deeper charge breaking ones. For the dark matter minimum, there was a clear demarcation for the regions where $\mathrm{CB}$ could occur, for which we have deduced a necessary and sufficient condition for full stability, but not so for the softly broken model. We conclude that CB global minima can indeed coexist, in some cases fairly frequently, with neutral minima and the potential for instability is therefore quite present.

\section{ACKNOWLEDGEMENTS}

This work is supported by Fundação para a Ciência e a Tecnologia (FCT, Portugal) through the projects UIDB/00777/2020, UIDP/00777/2020, UIDB/00618/2020, CERN/FIS-PAR/0004/2019, CERN/FISPAR/0014/2019, PTDC/FIS-PAR/29436/2017 and by HARMONIA project's contract UMO-2015/18/M/ST2/00518. The work of B.L.G. is supported by the FCT grant SFRH/BD/139165/2018.

\section{References}

[1] P.M. Ferreira and B.L. Gonçalves. Stability of neutral minima against charge breaking in the Higgs triplet model. JHEP, 02:182, 2020.

[2] Georges Aad et al. Observation of a new particle in the search for the Standard Model Higgs boson with the ATLAS detector at the LHC. Phys. Lett., B716:1-29, 2012.

[3] Serguei Chatrchyan et al. Observation of a new boson at a mass of $125 \mathrm{GeV}$ with the CMS experiment at the LHC. Phys. Lett., B716:30-61, 2012.

[4] Georges Aad et al. Combined Measurement of the Higgs Boson Mass in $p p$ Collisions at $\sqrt{s}=7$ and 8 TeV with the ATLAS and CMS Experiments. Phys. Rev. Lett., 114:191803, 2015.

[5] Georges Aad et al. Measurements of the Higgs boson production and decay rates and constraints on its couplings from a combined ATLAS and CMS analysis of the LHC pp collision data at $\sqrt{s}=7$ and $8 \mathrm{TeV}$. JHEP, 08:045, 2016.

[6] T. D. Lee. A Theory of Spontaneous T Violation. Phys. Rev., D8:1226-1239, 1973.

[7] G. C. Branco, P. M. Ferreira, L. Lavoura, M. N. Rebelo, Marc Sher, and Joao P. Silva. Theory and phenomenology of two-Higgs-doublet models. Phys. Rept., 516:1-102, 2012

[8] Rabindra N. Mohapatra and Goran Senjanovic. Neutrino Mass and Spontaneous Parity Nonconservation. Phys. Rev. Lett., 44:912, 1980. [,231(1979)].

[9] W. Konetschny and W. Kummer. Nonconservation of Total Lepton Number with Scalar Bosons. Phys. Lett., 70B:433-435, 1977.

[10] M. Magg and C. Wetterich. Neutrino Mass Problem and Gauge Hierarchy. Phys. Lett., 94B:61-64, 1980.

[11] T. P. Cheng and Ling-Fong Li. Neutrino Masses, Mixings and Oscillations in SU(2) x U(1) Models of Electroweak Interactions. Phys. Rev., D22:2860, 1980. 
[12] J. Schechter and J. W. F. Valle. Neutrino Masses in SU(2) x U(1) Theories. Phys. Rev., D22:2227, 1980.

[13] George Lazarides, Q. Shafi, and C. Wetterich. Proton Lifetime and Fermion Masses in an SO(10) Model. Nucl. Phys., B181:287-300, 1981.

[14] A. Arhrib, R. Benbrik, M. Chabab, G. Moultaka, M. C. Peyranere, L. Rahili, and J. Ramadan. The Higgs Potential in the Type II Seesaw Model. Phys. Rev., D84:095005, 2011.

[15] A. Arhrib, R. Benbrik, M. Chabab, G. Moultaka, and L. Rahili. Higgs boson decay into 2 photons in the type II Seesaw Model. JHEP, 04:136, 2012.

[16] Shinya Kanemura and Hiroaki Sugiyama. Dark matter and a suppression mechanism for neutrino masses in the Higgs triplet model. Phys. Rev., D86:073006, 2012.

[17] Mayumi Aoki, Shinya Kanemura, Mariko Kikuchi, and Kei Yagyu. Renormalization of the Higgs Sector in the Triplet Model. Phys. Lett., B714:279-285, 2012.

[18] Mayumi Aoki, Shinya Kanemura, Mariko Kikuchi, and Kei Yagyu. Radiative corrections to the Higgs boson couplings in the triplet model. Phys. Rev., D87(1):015012, 2013.

[19] Xun-Jie Xu. Minima of the scalar potential in the type II seesaw model: From local to global. Phys. Rev., D94(11):115025, 2016.

[20] J. Velhinho, R. Santos, and A. Barroso. Tree level vacuum stability in two Higgs doublet models. Phys. Lett., B322:213-218, 1994.

[21] P. M. Ferreira, R. Santos, and A. Barroso. Stability of the tree-level vacuum in two Higgs doublet models against charge or CP spontaneous violation. Phys. Lett., B603:219-229, 2004. [Erratum: Phys. Lett.B629,114(2005)].

[22] A. Barroso, P. M. Ferreira, and R. Santos. Charge and CP symmetry breaking in two Higgs doublet models. Phys. Lett., B632:684-687, 2006.

[23] C. C. Nishi. CP violation conditions in N-Higgs-doublet potentials. Phys. Rev., D74:036003, 2006. [Erratum: Phys. Rev.D76,119901(2007)].

[24] M. Maniatis, A. von Manteuffel, O. Nachtmann, and F. Nagel. Stability and symmetry breaking in the general two-Higgs-doublet model. Eur. Phys. J., C48:805-823, 2006.

[25] I. P. Ivanov. Minkowski space structure of the Higgs potential in 2HDM. Phys. Rev., D75:035001, 2007. [Erratum: Phys. Rev.D76,039902(2007)].

[26] A. Barroso, P. M. Ferreira, and R. Santos. Neutral minima in two-Higgs doublet models. Phys. Lett., B652:181-193, 2007.

[27] Celso C. Nishi. The Structure of potentials with N Higgs doublets. Phys. Rev., D76:055013, 2007.

[28] M. Maniatis, A. von Manteuffel, and O. Nachtmann. CP violation in the general two-Higgs-doublet model: A Geometric view. Eur. Phys. J., C57:719-738, 2008.

[29] Igor P. Ivanov. Minkowski space structure of the Higgs potential in 2HDM. II. Minima, symmetries, and topology. Phys. Rev., D77:015017, 2008.

[30] M. Maniatis, A. von Manteuffel, and O. Nachtmann. A New type of CP symmetry, family replication and fermion mass hierarchies. Eur. Phys. J., C57:739-762, 2008.

[31] C. C. Nishi. Physical parameters and basis transformations in the Two-Higgs-Doublet model. Phys. Rev., D77:055009, 2008.

[32] M. Maniatis and O. Nachtmann. On the phenomenology of a two-Higgs-doublet model with maximal CP symmetry at the LHC. JHEP, 05:028, 2009.

[33] P. M. Ferreira, M. Maniatis, O. Nachtmann, and Joao P. Silva. CP properties of symmetry-constrained two-Higgs-doublet models. JHEP, 08:125, 2010. 